\title{
Relation of kidney function and homocysteine in patients with hypothyroidism
}

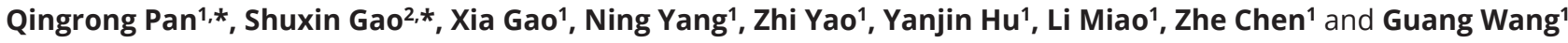 \\ 'Department of Endocrinology, Beijing Chao-yang Hospital, Capital Medical University, Beijing, China \\ 2Department of General Practice, Cangzhou Central Hospital, Cangzhou, Hebei Province, China
}

Correspondence should be addressed to Z Chen or G Wang: 2227157457@qq.com or drwg6688@163.com

*(Q Pan and S Gao contributed equally to this work)

\begin{abstract}
Objective: It has been found that both serum homocysteine (Hcy) and serum creatinine levels were increased in hypothyroidism patients. The aim of this study was to investigate the correlation between serum Hcy and kidney function in patients with subclinical hypothyroidism or hypothyroidism.

Methods: A total of 448 subjects were enrolled and divided into three groups: hypothyroidism ( $n=129)$, subclinical hypothyroidism $(n=141)$, and control group $(n=168)$. Anthropometric information, metabolic parameters, serum Hcy and creatinine levels, and estimated glomerular filtration rate (eGFR) were analyzed.

Results: Compared with healthy subjects, patients with subclinical hypothyroidism or hypothyroidism had significantly higher serum Hcy and creatinine levels and lower eGFR level (all $P<0.001$ ). Serum Hcy was negatively correlated with eGFR in subclinical hypothyroidism patients $(r=-0.220, P=0.009)$, and in hypothyroidism patients $(r=-0.422, P<0.001)$. After adjusting for age, sex and BMI, eGFR was still significantly correlated with serum Hcy in subclinical hypothyroidism or hypothyroidism patients (both $P<0.05$ ). Levothyroxine treatment resulted in significantly decreased $\mathrm{Hcy}$ and increased eGFR in hypothyroidism patients (both $P<0.001$ ). The decrease in Hcy was correlated with the increased eGFR after treatment $(P=0.001)$.

Conclusion: Serum Hcy was negatively correlated with eGFR in subclinical hypothyroidism or hypothyroidism patients. After levothyroxine treatment, a correlation was found between the decrease in serum Hcy and the increase in eGFR in hypothyroidism patients.
\end{abstract}

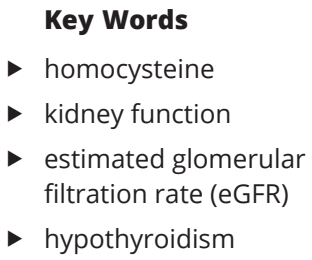

Endocrine Connections (2021) 10, 502-510

\section{Introduction}

Subclinical hypothyroidism and clinical hypothyroidism, two commonly encountered clinical conditions, can induce various metabolic changes (1) and increase the risk of cardiovascular diseases $(2,3)$. Recent researches have shown that both subclinical hypothyroidism and hypothyroidism were associated with elevated serum creatinine, decreased estimated glomerular filtration rate (eGFR) and increased risk of chronic kidney disease (CKD) $(4,5,6,7)$. Moreover, the recent evidence from a mendelian randomization study demonstrated a directional association from hypothyroidism to decreased eGFR and increased CKD (8).

Homocysteine (Hcy) is a sulfur-containing amino acid, which is the intermediate product of methionine demethylation. Hyperhomocysteinemia is a well-known independent risk factor for atherosclerosis and coronary heart disease $(9,10,11)$. Recent investigations and our previous studies demonstrated that the blood Hcy

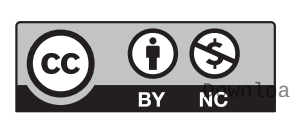

This work is licensed under a Creative Commons Attribution-NonCommercial 4.0 International License. ded from Bioscientifica.com at 04/26/2023 10:41:19AM 
level was increased in patients with both subclinical hypothyroidism and hypothyroidism $(12,13,14)$. However, the potential pathophysiological mechanism underlying the increase of Hcy level in thyroid hormone deficiency state has not been fully elucidated. It has been speculated that the increase of Hcy was at least partially due to the decrease of renal function (15), because of the evidence that Hcy was negatively correlated with kidney function in general population $(16,17,18)$. However, it is unknown whether serum Hcy level is also associated with kidney function in subclinical hypothyroidism or hypothyroidism patients.

Therefore, the aim of this study was to investigate the correlation between serum Hcy and kidney function in patients with subclinical hypothyroidism and hypothyroidism. Furthermore, we investigated the treatment effects of levothyroxine (L-T4) on serum Hcy level and kidney function in hypothyroidism patients, and the association between the changes of Hcy and kidney function after L-T4 treatment.

\section{Materials and methods}

\section{Subjects}

A total of 305 patients with subclinical hypothyroidism or hypothyroidism were initially enrolled at the outpatient clinic of the endocrinology department in Beijing Chao-yang Hospital from January 2018 to July 2020. Hypothyroidism was defined as an elevated thyrotropin (TSH) level concomitant with a decreased thyroid hormone. Subclinical hypothyroidism was defined as an increased TSH level when concentrations of thyroid hormones were within their normal ranges. The normal values for TSH, free triiodothyronine (FT3) and free thyroxine (FT4) were $0.55-4.78 \mu \mathrm{IU} / \mathrm{mL}, 2.3-4.2 \mathrm{pg} / \mathrm{mL}$ and $8.9-17.6 \mathrm{pg} / \mathrm{mL}$, respectively.

Exclusion criteria were as follows: patients under treatment with thyroxine, or anti-thyroid, patients with cardiovascular disease, hypertension, diabetes, hepatic diseases, kidney diseases, infection diseases, malignant tumors or systemic autoimmune diseases. Therefore, 35 patients were excluded. The final study cohort consisted of 141 subclinical hypothyroidism patients and 129 hypothyroidism patients. One hundred and sixty eight healthy euthyroid subjects who were seeking routine medical health checkups at the physical examination center of Beijing Chao-yang Hospital, matched for age and sex of the patients, were included.
We defined serum $\mathrm{Hcy} \geq 15 \quad \mu \mathrm{mol} / \mathrm{L}$ as hyperhomocysteinemia. All participants were divided into six subgroups according to the presence or absence of hyperhomocysteinemia for further analysis.

In the hypothyroidism group, all patients were given $\mathrm{L}-\mathrm{T} 4$ treatment. The treatment dose started from $50 \mu \mathrm{g} / \mathrm{day}$. For dose adjustment, thyroid function was measured every 4 weeks. Among 129 patients with hypothyroidism, 58 patients were followed for 34 months -4 months until thyroid function turned normal and received serum Hcy and renal function reexamination.

This study was approved by the Ethics Committee of Beijing Chao-yang Hospital (No. 2012-ke-97) and performed in accordance with the Helsinki Declaration. All study participants provided a written informed consent.

\section{Measurement}

Anthropometric information, including age, sex, body height and weight, was collected. BMI was calculated as weight $/$ height $^{2}\left(\mathrm{~kg} / \mathrm{m}^{2}\right)$. Serum samples were obtained after overnight fasting. Creatinine, urea nitrogen, albumin, triglyceride, total cholesterol (TC), HDL-C, low density lipoprotein cholesterol (LDL-C) and glucose levels were measured on a Siemens ADVIA 2400 automatic biochemical analyzer. The serum total Hcy was measured by commercially available enzyme cycling assay kits (Biosino Bio-Technology And Science Inc, Beijing, China). Serum thyroid hormones were measured by direct chemiluminescent technology (Siemens Healthcare Diagnostics In). eGFR ( $\left.\mathrm{mL} / \mathrm{min} / 1.73 \mathrm{~m}^{2}\right)$ was calculated according to the Chronic Kidney Disease Epidemiology Collaboration (CKD-EPI) equation as following (19): for female, if serum creatinine $\leq 0.7 \mathrm{mg} / \mathrm{dL}$, $\mathrm{eGFR}=144 \times(\text { serum creatinine }(\mathrm{mg} / \mathrm{dL}) / 0.7)^{-0.329} \times$ $(0.993)^{\text {age}}$; if serum creatinine $>0.7 \mathrm{mg} / \mathrm{dL}$, eGFR $=144$ $\times$ (serum creatinine $(\mathrm{mg} / \mathrm{dL}) / 0.7)^{-1.209} \times(0.993)^{\text {age}}$; for male, if serum creatinine $(\mathrm{mg} / \mathrm{dL}) \leq 0.9$, eGFR $=141 \times$ (serum creatinine $(\mathrm{mg} / \mathrm{dL}) / 0.9)^{-0.411} \times(0.993)^{\text {age}}$; if serum creatinine $>0.9 \mathrm{mg} / \mathrm{dL}$, eGFR $=141 \times$ (serum creatinine $(\mathrm{mg} / \mathrm{dL}) / 0.9)^{-1.209} \times(0.993)^{\mathrm{age}}$.

\section{Statistical analysis}

The statistical analysis was performed using IBM SPSS statistical version 21.0. Continuous variables were showed as mean \pm s.D. or median (lower and upper quartiles). Categorical variables were shown as number (percent). Continuous variable with normal distribution was compared by ANOVA and Bonferroni 
post hoc analysis; variable with skewed distribution, including triglyceride, Hcy, creatinine and eGFR was compared using nonparametric Kruskal-Wallis rank test and Mann-Whitney $U$-test. Categorical variables were compared with the chi-square between groups. Changes in parameters after treatment were analyzed using the paired $t$-test or the nonparametric Wilcoxon rank test. Univariate associations were tested by Spearman correlation. Multiple stepwise linear regression analyses were used to examine whether there was an independent association between Hcy and eGFR after adjusting confounding factors. The dependent variable was serum Hcy; the independent variables were eGFR, age, sex and BMI. Hcy and eGFR were $\log _{10}$ transformed before included in the regression model, because of their skewed distribution. To investigate whether the correlations between serum Hcy and eGFR were different among healthy subjects, subclinical hypothyroidism patients and hypothyroidism patients, we performed the interaction test between eGFR and groups using the ANOVA. A value of $P<0.05$ was considered to be statistically significant.

\section{Results}

Clinical characteristics of healthy controls, patients with subclinical hypothyroidism, and patients with hypothyroidism

The clinical characteristics of healthy controls, patients with subclinical hypothyroidism, and patients with hypothyroidism were listed in Table 1. Sex, age, BMI, fasting blood glucose, and albumin levels were similar among the three groups. Serum TC and HDL-C levels were significantly higher in hypothyroidism group than those in the subclinical hypothyroidism and control groups (all $P<0.05$ ). Serum triglyceride was significantly higher in subclinical hypothyroidism and hypothyroidism groups than that in healthy controls $(P<0.05)$. Serum LDL-C was significantly higher in hypothyroidism group than that in control group $(P<0.05)$. Compared with healthy controls, the patients with subclinical hypothyroidism and those with hypothyroidism had significantly higher serum Hcy, urea nitrogen, and creatinine levels, and significantly lower eGFR level (all $P<0.05$ ). Moreover, compared with subclinical hypothyroidism patients, hypothyroidism patients had significantly higher serum Hcy and creatinine levels, and significantly lower eGFR levels (all $P<0.05)$.

\section{Clinical characteristics of subgroups with and without hyperhomocysteinemia}

Clinical parameters of hyperhomocysteinemia subgroups and normal homocysteine subgroups were shown in Table 2. Compared with those in subgroups with normal homocysteine, subjects in subgroups with hyperhomocysteinemia were more likely to be male, had significantly higher serum triglyceride and creatinine, and significantly lower eGFR levels as compared with the corresponding subgroups without hyperhomocysteinemia (all $P<0.05$ ).

Table 1 Clinical characteristics of healthy controls, patients with subclinical hypothyroidism and patients with hypothyroidism.

\begin{tabular}{lcc}
\hline Parameters & & Control $(n=168)$ \\
\cline { 1 - 1 } Age, years & & $41.39 \pm 9.97$ \\
Sex, male $(\%)$ & $22(13.10)$ \\
BMl, km/m² & & $23.73 \pm 4.47$ \\
Albumin, g/L & $45.34 \pm 2.46$ \\
FBG, mmol/L & $5.09 \pm 0.59$ \\
TC, mmol/L & $4.89 \pm 0.87$ \\
HDL-C, mmol/L & $1.37 \pm 0.33$ \\
LDL-C, mmol/L & $2.86 \pm 0.72$ \\
Triglyceride, mmol/L & $1.10(0.84-1.48)$ \\
Hcy, $\mu \mathrm{mol} / \mathrm{L}$ & & $10.0(8.0-11.0)$ \\
Urea nitrogen, mmol/L & $4.52 \pm 1.24$ \\
Creatinine, $\mu \mathrm{mol} / \mathrm{L}$ & $52.35(48.32-56.95)$ \\
eGFR, mL/min/1.73 m ${ }^{2}$ & $115.66(108.35-120.90)$ \\
\hline
\end{tabular}

\begin{tabular}{c} 
Subclinical hypothyroidism $(n=141)$ \\
\hline $41.76 \pm 11.01$ \\
$22(15.60)$ \\
$24.37 \pm 4.22$ \\
$46.15 \pm 4.17$ \\
$5.06 \pm 0.55$ \\
$5.04 \pm 1.04$ \\
$1.32 \pm 0.32$ \\
$3.04 \pm 0.85$ \\
$1.26(0.91-1.86)^{*}$ \\
$11.0(10.0-14.0)^{\star *}$ \\
$4.88 \pm 1.04^{*}$ \\
$59.30(52.4-67.35)^{* *}$ \\
$108.68(99.45-119.03)^{* *}$
\end{tabular}

\begin{tabular}{c}
\hline Hypothyroidism $(n=129)$ \\
\hline $39.55 \pm 11.72$ \\
$18(13.95)$ \\
$24.80 \pm 4.61$ \\
$46.14 \pm 3.15$ \\
$5.03 \pm 0.55$ \\
$5.57 \pm 1.63^{* *+\dagger}$ \\
$1.49 \pm 0.46^{* \dagger \dagger}$ \\
$3.21 \pm 1.15^{* *}$ \\
$1.24(0.88-2.19)^{*}$ \\
$13.0(11.0-16.0)^{* *+\dagger}$ \\
$4.92 \pm 1.36^{*}$ \\
$62.70(53.85-77.45)^{* * \dagger}$ \\
$103.92(85.85-117.90)^{* * \dagger}$
\end{tabular}

\begin{tabular}{c}
\hline \multicolumn{1}{c}{$\boldsymbol{P}$} \\
\hline 0.112 \\
0.735 \\
0.112 \\
0.21 \\
0.061 \\
$<0.001$ \\
$<0.001$ \\
0.004 \\
0.016 \\
$<0.001$ \\
0.012 \\
$<0.001$ \\
$<0.001$ \\
\hline
\end{tabular}

Data were expressed as the means \pm S.D., medians (upper and lower quartiles), or $n$ (\%).

${ }^{*} P<0.05$ significantly different compared with control group; ${ }^{\dagger} P<0.05$ significantly different compared with subclinical hypothyroidism group; ${ }^{* *} P<0.01$ significantly different compared with control group; ${ }^{\dagger \dagger} P<0.01$ significantly different compared with hypothyroidism group.

eGFR, estimated glomerular filtration rate; FBG, fasting blood glucose; Hcy, homocysteine; HDL-C, high-density lipoprotein cholesterol; LDL-C, low-density lipoprotein cholesterol; TC, total cholesterol.

https://ec.bioscientifica.com

https://doi.org/10.1530/EC-21-0069 (c) 2021 The authors Published by Bioscientifica Ltd

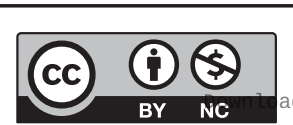

This work is licensed under a Creative Commons Attribution-NonCommercial 4.0 International License. ded from Bioscientifica.com at 04/26/2023 10:41:19AM 


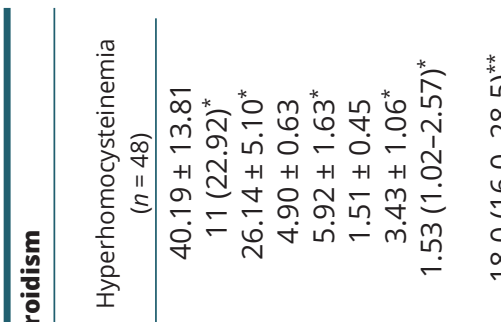

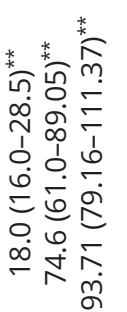

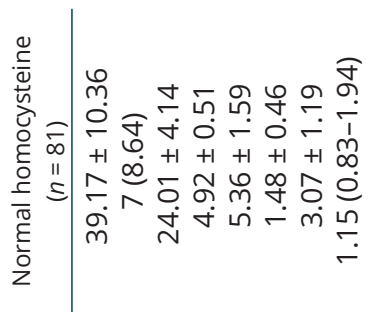

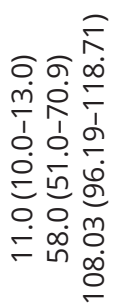

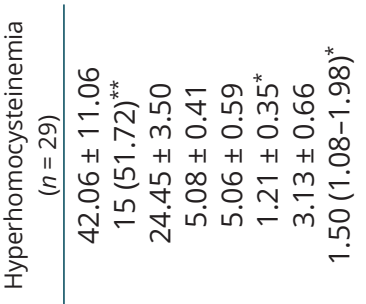

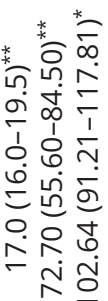

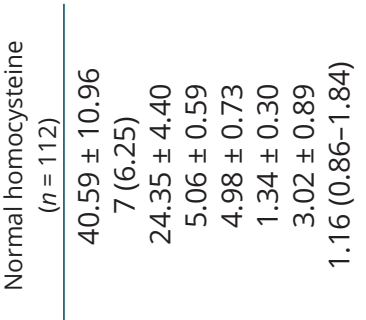

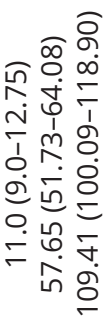

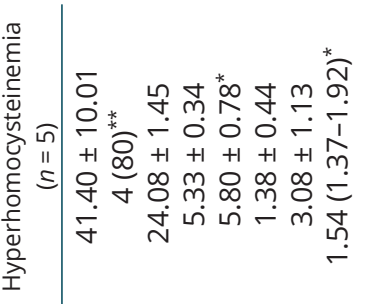

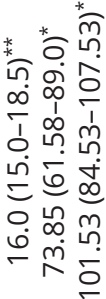

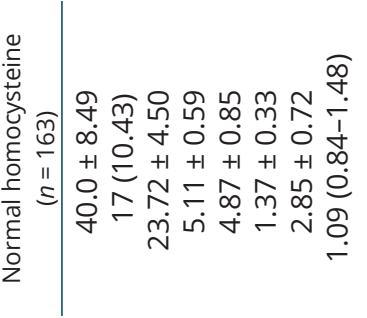
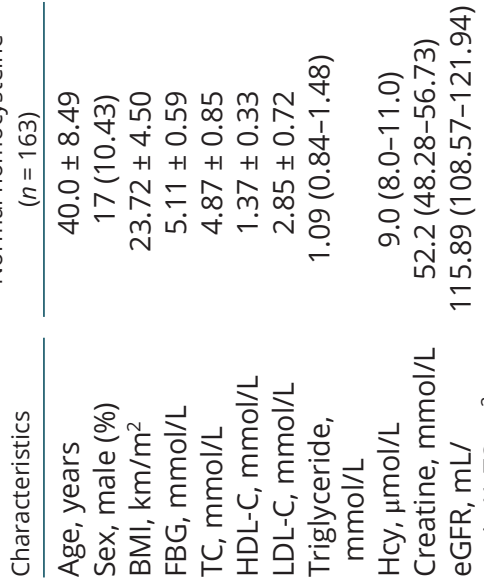

है है

है

N

\section{Correlation between eGFR and serum Hcy level at baseline}

Spearman's correlation analyses (Fig. 1) showed that serum Hcy level was negatively correlated with eGFR not only in healthy controls $(\mathrm{r}=-0.523, P<0.001)$ but also in subclinical hypothyroidism patients $(\mathrm{r}=-0.220$, $P=0.009)$ and hypothyroidism patients $(\mathrm{r}=-0.422$, $P<0.001$ ). Multiple linear regression analyses (Table 3 ) revealed that after adjusting for age, sex and BMI, eGFR was still significantly correlated with serum Hcy in control group ( $\beta=-0.187, P=0.024$ ), subclinical hypothyroidism group ( $\beta=-0.287, P=0.010$ ), and hypothyroidism group $(\beta=-0.519, P<0.001)$. There was no significant interaction between eGFR and the groups $(\mathrm{F}=1.270, P=0.282)$.

\section{The changes in eGFR and serum Hcy level in hypothyroidism group after L-T4 treatment}

Among hypothyroidism patients, 58 of the hypothyroidism patients were followed-up. After 3-4 months' treatment of L-T4, triglyceride, TC, HDL-C and LDL-C were significantly decreased (all $P<0.05$ ), serum Hcy level was significantly decreased $(P<0.001)$ and eGFR was significantly increased $(P<0.001$, Table 4 , Fig. 2$)$. The mediums of the changes of serum Hcy $(\Delta$ Hcy $)$ and eGFR ( $\triangle$ eGFR) were $-3.0(-5.0 \sim-2.0) \mu \mathrm{mol} / \mathrm{L}$ and 14.26 (3.78 26.61) $\mathrm{mL} / \mathrm{min} / 1.73 \mathrm{~m}^{2}$, respectively. There was a significant correlation between $\Delta$ Hcy and $\triangle$ eGFR in hypothyroidism patients $(\mathrm{r}=-0.423, P=0.001$, Fig. 3$)$.

\section{Discussion}

In the present study, we found that the patients with subclinical hypothyroidism and those with hypothyroidism had significantly higher serum Hcy and creatinine levels, and lower eGFR level as compared with healthy subjects. Moreover, serum Hcy was negatively correlated with eGFR in subclinical hypothyroidism and hypothyroidism patients. Multiple linear regression analyses revealed that eGFR was still significantly correlated with serum Hcy after adjusting for age, sex and BMI. After 4-5 months' treatment of L-T4, serum Hcy level was significantly decreased and eGFR was significantly increased in hypothyroidism patients. The changes of serum Hcy $(\Delta$ Hcy) and eGFR ( $\triangle$ eGFR) were correlated.

Previous studies have shown that both hypothyroidism and subclinical hypothyroidism were associated with elevated serum creatinine, decreased 

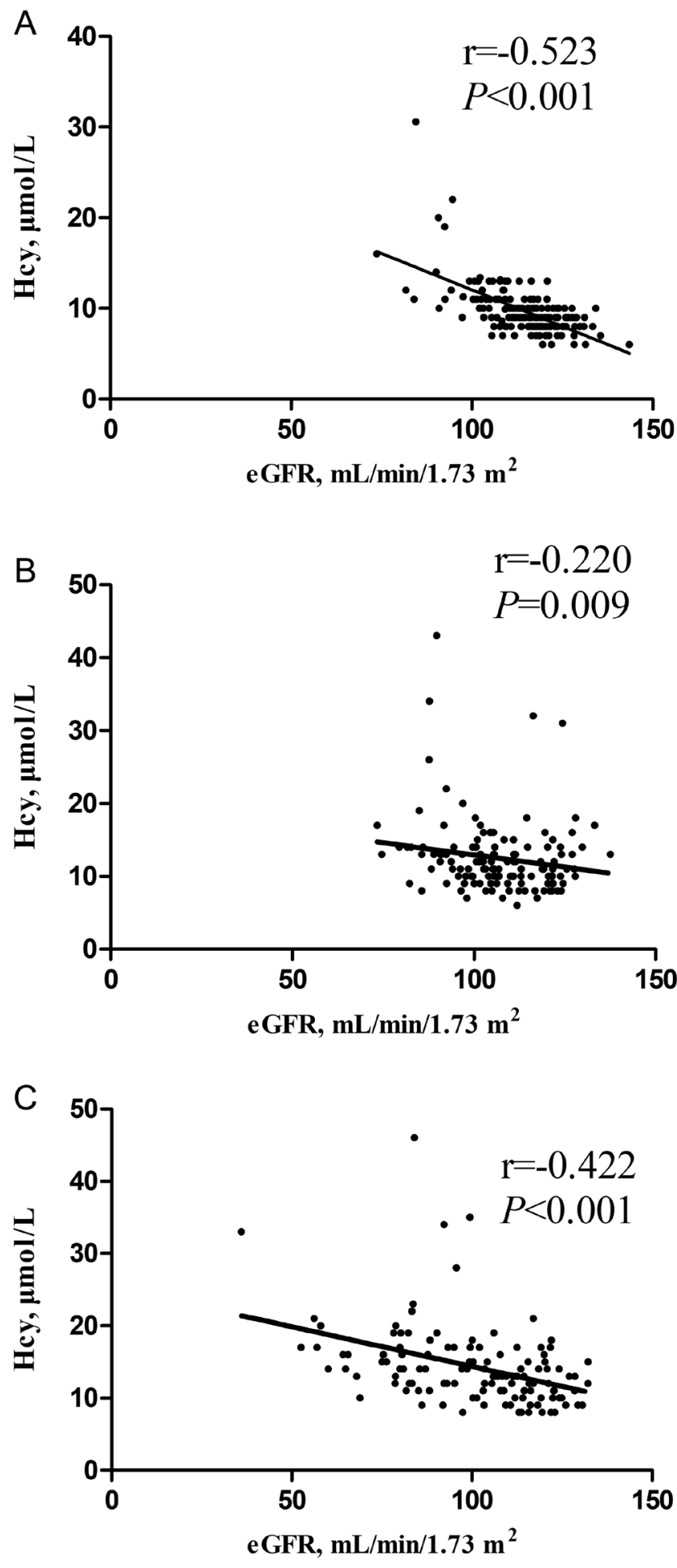

Figure 1

Correlations between serum Hcy levels and eGFR in healthy controls (A), subclinical hypothyroidism patients (B), and hypothyroidism patients (C).
eGFR and increased risk of CKD $(4,5,6,7)$. Recent investigations and our previous studies demonstrated that Hcy level was increased in patients with hypothyroidism and those with subclinical hypothyroidism $(12,13,14)$. Our results of elevated serum Hcy and lower eGFR level in subclinical hypothyroidism and hypothyroidism groups were consistent with these previous findings.

A mendelian randomization study revealed a direction of the association of hypothyroidism to decreased eGFR and increased CKD, but not vice versa (8). In addition, the potential mechanisms connecting the thyroid hormone deficiency to a decrease in kidney function have been fully discussed. It has been demonstrated that thyroid hormone deficiency could induce direct and indirect renal effects, including a reduction in glomeruli size, a decrease of cardiac output, a change in vascular resistance, and changes in sodium and water homeostasis $(20,21)$. In contrast, the mechanisms for the increased Hcy level in hypothyroidism were not clearly understood yet. It has been demonstrated that the kidney was involved in the metabolic clearance of Hcy (22). In addition, a negative relationship between blood Hcy level and renal function has been found in general population $(16,17$, 18). Therefore, there was a hypothesis that the decrease of eGFR in hypothyroidism at least partially contributed to the Hcy elevation (15). The correlations between serum Hcy and eGFR in subclinical hypothyroidism and hypothyroidism patients revealed by our study provided additional information for this hypothesis.

The underlying mechanisms and the cause direction of connection between renal function and Hcy have been previously debated. More than $75 \%$ of total serum Hcy was bound to proteins, mainly albumin, and only a minor percentage of Hcy was eliminated through the glomerular filter (23). Metabolic processing removed the majority of Hcy in the body, with kidney playing an important role (22). Several studies showed that both animal and human kidneys contained enzymes involved in Hcy-metabolism, including betainehomocysteine S-methyltransferase, cystathionine betasynthase and 5-methyltetrahydrofolate-homocysteine methyltransferase $(24,25)$. In addition, the kidney played a major role in the removal of Hcy-related compounds from circulation (such as glutathione, cysteine-glycine, S-adenosylmethionine, and S-adenosylhomocysteine) $(22,26)$. Thus, the reduced kidney function in thyroid hormone deficiency state probably could cause a decrease in Hcy removal from the body.

On the other hand, a series of evidence indicated that serum Hcy might contribute to a decline in kidney
This work is licensed under a Creative Commons Attribution-NonCommercial 4.0 International License. ded from Bioscientifica.com at 04/26/2023 10:41:19AM via free access 
Table 3 The correlations between eGFR and serum Hcy in control group, subclinical hypothyroidism group and hypothyroidism group after adjusting for age, sex and BMI.

\begin{tabular}{l} 
Groups \\
\hline Control \\
Subclinical hypothyroidism \\
Hypothyroidism
\end{tabular}

\begin{tabular}{l}
\hline Independent variable \\
\hline $\log 10(\mathrm{eGFR})$ \\
$\log 10(\mathrm{eGFR})$ \\
$\log 10(\mathrm{eGFR})$ \\
\hline
\end{tabular}

\begin{tabular}{ccc}
\hline \multicolumn{3}{c}{ Unstandardized co } \\
\hline B & & SE \\
\hline-0.296 & & 0.132 \\
-0.952 & & 0.335 \\
-0.828 & & 0.143 \\
\hline
\end{tabular}

\begin{tabular}{l} 
coefficients \\
\hline$-0.470 \sim-0.037$ \\
$-1.614 \sim-0.290$ \\
$-1.111 \sim-0.545$
\end{tabular}

\begin{tabular}{crr}
\hline $\begin{array}{c}\text { Standardized } \\
\text { coefficients }(\beta)\end{array}$ & \multicolumn{1}{c}{$\boldsymbol{P}$} \\
\cline { 1 - 1 }-0.187 & & 0.024 \\
-0.287 & & 0.010 \\
-0.519 & & $<0.001$ \\
\hline
\end{tabular}

The dependent variable was serum Hcy; the independent variables were eGFR, age, sex and BMI. Hcy and eGFR were log10 transformed before included in the linear regression model, because of their skewed distribution.

eGFR, estimated glomerular filtration rate; Hcy, homocysteine.

function. Hcy could cause nephrotoxicity by reducing serum and tissue adenosine levels (27), promoting endothelial cell, mesangial cell and podocyte injury through inflammation and oxidative stress (28, 29). Framingham offspring study showed that Hcy was an independent risk factor for future CKD or microalbuminuria (30). Other prospective studies demonstrated that subjects with hyperhomocysteinemia had a higher odds ratio of renal function decline and future incidence of CKD $(31,32,33,34)$. However, available interventional studies have provided conflicting results of renal outcomes. The subgroup analysis of Chinese primary stroke prevention trial (CSPPT) demonstrated that folic acid supplementation significantly reduced the decline of eGFR and delayed the progress of CKD (35). In contrast, Homocysteinemia in kidney and end stage renal disease (HOST) trial showed that adding folic acid and vitamin B12 did not affect the time to dialysis in patients with advanced CKD (36). Another randomized controlled trial demonstrated that high doses of folic acid and vitamin B compared with placebo did not delay CKD progression in patients with diabetic nephropathy (37). One possible explanation for the beneficial effect of folic acid in the Chinese study was that, compared with studies in other countries, the Chinese study was conducted in a region without grain folic acid fortification. The above evidence, combined with our finding of correlation between serum Hcy and eGFR in subclinical hypothyroidism and hypothyroidism patients, suggested that probably there was a vicious circle between Hcy elevation and renal function impairment, which further increased the correlation between blood homocysteine and eGFR, not only in healthy subjects but also in subclinical hypothyroidism and hypothyroidism patients. We did not find a significant interaction between eGFR and different groups. It indicated that the correlation between Hcy and eGFR was similar among healthy subjects, subclinical hypothyroidism patients and hypothyroidism patients. The result of the decreased eGFR and elevated Hcy in hypothyroidism, which could be reversed by L-T4 treatment, suggested that patients with hypothyroidism should be treated as early as possible.

The present study has several limitations. First, the intakes of folic acid or vitamin B were not recorded and serum folic acid was not measured, thus these parameters could not be included in the analyses. Secondly, other

Table 4 Comparison of thyroid function, serum lipid, creatinine, Hcy and eGFR levels in hypothyroidism patients before and after L-T4 treatment $(n=58)$.

\begin{tabular}{l}
\hline Parameters \\
\hline FT3 \\
FT4 \\
TSH \\
TC, $\mathrm{mmol} / \mathrm{L}$ \\
$\mathrm{HDL}-\mathrm{C}, \mathrm{mmol} / \mathrm{L}$ \\
$\mathrm{LDL}-\mathrm{C}, \mathrm{mmol} / \mathrm{L}$ \\
Triglyceride, $\mathrm{mmol} / \mathrm{L}$ \\
Hcy, $\mu \mathrm{mol} / \mathrm{L}$ \\
Creatinine, $\mu \mathrm{mol} / \mathrm{L}$ \\
eGFR, $\mathrm{mL} / \mathrm{min} / 1.73 \mathrm{~m}^{2}$
\end{tabular}

\begin{tabular}{c}
\hline Baseline $(n=168)$ \\
\hline $2.25 \pm 0.90$ \\
$0.73 \pm 0.33$ \\
$43.84(7.29-100.0)$ \\
$5.81 \pm 1.57$ \\
$1.52 \pm 0.44$ \\
$3.36 \pm 1.07$ \\
$1.38(1.07-2.29)$ \\
$14.0(12.0-17.0)$ \\
$71.9(59.78-85.65)$ \\
$94.55(80.86-107.19)$
\end{tabular}

\begin{tabular}{c}
\hline After L-t4 treatment $(n=141)$ \\
\hline $2.87 \pm 0.38$ \\
$1.23 \pm 0.22$ \\
$2.28(1.44-3.59)$ \\
$4.60 \pm 1.04$ \\
$1.32 \pm 0.38$ \\
$2.80 \pm 0.76$ \\
$1.28(0.97-1.72)$ \\
$11.0(9.0-14.0)$ \\
$57.05(50.23-68.70)$ \\
$112.02(103.66-118.26)$
\end{tabular}

\begin{tabular}{rr}
\hline \multicolumn{1}{c}{$\boldsymbol{P}$} \\
\hline$<0.001$ \\
$<0.001$ \\
$<0.001$ \\
$<0.001$ \\
$<0.001$ \\
$<0.001$ \\
0.007 \\
$<0.001$ \\
$<0.001$ \\
$<0.001$
\end{tabular}

eGFR, estimated glomerular filtration rate; FT3, free triiodothyronine; FT4, free thyroxine; Hcy, homocysteine; HDL-C, high-density lipoprotein cholesterol; LDL-C, low-density lipoprotein cholesterol; TC, total cholesterol; TSH, thyrotropin.

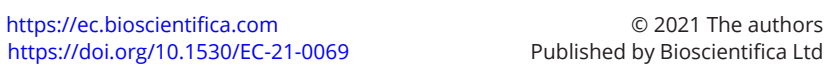



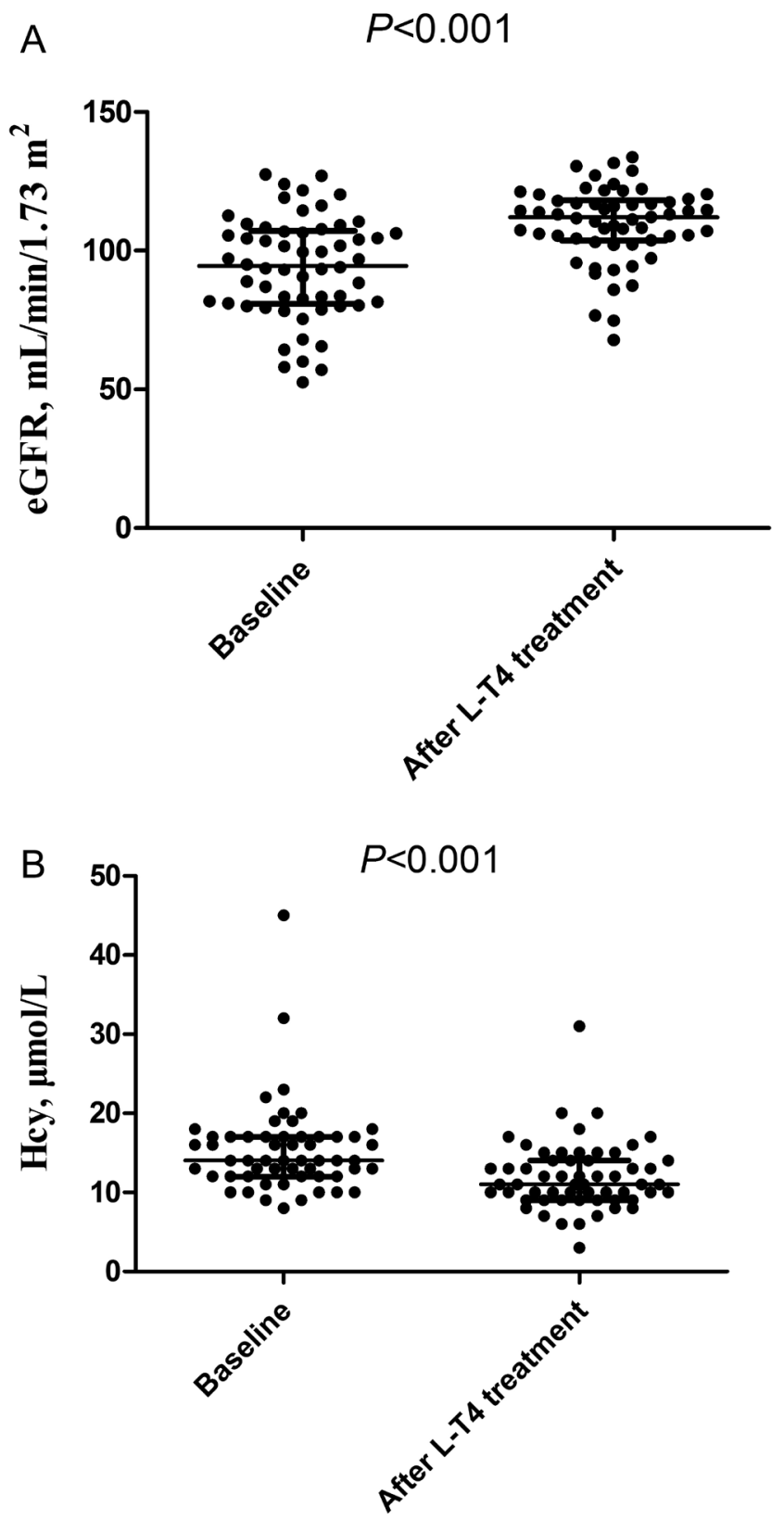

Figure 2

Comparison of eGFR (A) and serum Hcy (B) in hypothyroidism patients before and after L-T4 treatment $(n=58)$. Data were expressed as medium (upper/lower quartile).

renal markers such as urine albumin level and serum cystatin C, which might further support our findings, were not available. Thirdly, subclinical hypothyroidism patients have not been followed-up.

In conclusion, this study showed a negative association between serum Hcy and eGFR in subclinical hypothyroidism and overt hypothyroidism patients. After L-T4 treatment, a correlation was found between the decrease of serum Hcy ( $\Delta$ Hcy) and the increase

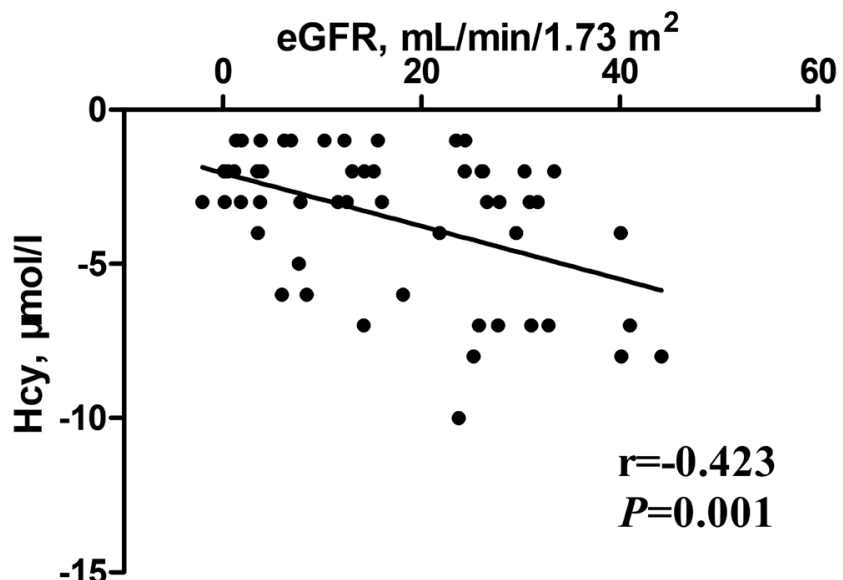

Figure 3

Correlation between the changes of serum Hcy $(\Delta \mathrm{Hcy})$ and eGFR ( $\triangle \mathrm{eGFR}$ ) after L-T4 treatment in hypothyroidism patients.

of eGFR ( $\Delta$ Hcy) in hypothyroidism patients. Our findings provided additional information to explain the underlying mechanisms of Hcy elevation in subclinical hypothyroidism and hypothyroidism patients.

Declaration of interest

The authors declare that there is no conflict of interest that could be perceived as prejudicing the impartiality of the research reported.

\section{Funding}

This study was funded by a grant from Beijing Key Laboratory of Metabolic Disorder Related Cardiovascular Disease (No. Dxwl2019op002) to Zhe Chen; and National Natural Science Foundation of China (No.81770792) to Guang Wang. The funder had no role in the study design, data collection and analysis, decision to publish, or preparation of the manuscript.

Author contribution statement

Q P and G W conceived and designed the study; Q P, S G, X G, N Y, L M, Z Y, Y H and Z C performed the study; S G collected and analyzed the data; Q P wrote the paper; Z C and G W read through and corrected the manuscript.

\section{References}

1 Gong N, Gao C, Chen X, Wang Y \& Tian L. Adipokine expression and endothelial function in subclinical hypothyroidism rats. Endocrine Connections 20187 295-304. (https://doi.org/10.1530/ EC-18-0007)

2 Klein I \& Danzi S. Thyroid disease and the heart. Circulation 2007116 1725-1735. (https://doi.org/10.1161/ CIRCULATIONAHA.106.678326)

3 Saif A, Mousa S, Assem M, Tharwat N \& Abdelhamid A. Endothelial dysfunction and the risk of atherosclerosis in overt and subclinical hypothyroidism. Endocrine Connections 20187 1075-1080. (https:// doi.org/10.1530/EC-18-0194)

4 Zhou JB, Li HB, Zhu XR, Song HL, Zhao YY \& Yang JK. Subclinical hypothyroidism and the risk of chronic kidney disease in T2D 
subjects: A case-control and dose-response analysis. Medicine 201796 e6519. (https://doi.org/10.1097/MD.0000000000006519)

5 Saini V, Yadav A, Arora MK, Arora S, Singh R \& Bhattacharjee J. Correlation of creatinine with TSH levels in overt hypothyroidism - a requirement for monitoring of renal function in hypothyroid patients? Clinical Biochemistry 201245 212-214. (https://doi. org/10.1016/j.clinbiochem.2011.10.012)

6 Asvold BO, Bjoro T \& Vatten LJ. Association of thyroid function with estimated glomerular filtration rate in a population-based study: the HUNT study. European Journal of Endocrinology 2011164 101-105. (https://doi.org/10.1530/EJE-10-0705)

7 Rosario P \& Calsolari MR. Subclinical hypothyroidism with $\mathrm{TSH}>7 \mathrm{mIU} / 1$ and $</=10 \mathrm{mIU} / 1$ and coronary artery disease. Hormone and Metabolic Research 202052 85-88. (https://doi. org/10.1055/a-1083-6509)

8 Ellervik C, Mora S, Ridker PM \& Chasman DI. Hypothyroidism and kidney function: A Mendelian randomization study. Thyroid 202030 365-379. (https://doi.org/10.1089/thy.2019.0167)

9 Kalra DK. Homocysteine and cardiovascular disease. Current Atherosclerosis Reports 20046 101-106. (https://doi.org/10.1007/ s11883-004-0097-3)

10 Guba SC, Fink LM \& Fonseca V. Hyperhomocysteinemia. An emerging and important risk factor for thromboembolic and cardiovascular disease. American Journal of Clinical Pathology 1996 106 709-722. (https://doi.org/10.1093/ajcp/106.6.709)

11 Stampfer MJ, Malinow MR, Willett WC, Newcomer LM, Upson B, Ullmann D, Tishler PV \& Hennekens $\mathrm{CH}$. A prospective study of plasma homocyst(e)ine and risk of myocardial infarction in US physicians. Journal of American Medical Association 1992268 877-881.

12 Morris MS, Bostom AG, Jacques PF, Selhub J \& Rosenberg IH. Hyperhomocysteinemia and hypercholesterolemia associated with hypothyroidism in the third US National Health and Nutrition Examination Survey. Atherosclerosis 2001155 195-200. (doi:10.1016/ s0021-9150(00)00537-2)

13 Yang N, Yao Z, Miao L, Liu J, Gao X, Fan H, Hu Y, Zhang H, $\mathrm{Xu} \mathrm{Y}, \mathrm{Qu} \mathrm{A}$, et al. Novel clinical evidence of an association between homocysteine and insulin resistance in patients with hypothyroidism or subclinical hypothyroidism. PLOS ONE 201510 e0125922. (https://doi.org/10.1371/journal.pone.0125922)

14 Dong X, Yao Z, Hu Y, Yang N, Gao X, Xu Y \& Wang G. Potential harmful correlation between homocysteine and low-density lipoprotein cholesterol in patients with hypothyroidism. Medicine 201695 e4291. (https://doi.org/10.1097/MD.0000000000004291)

15 Orzechowska-Pawilojc A, Sworczak K, Lewczuk A \& Babinska A. Homocysteine, folate and cobalamin levels in hypothyroid women before and after treatment. Endocrine Journal 200754 471-476. (https://doi.org/10.1507/endocrj.k06-112)

16 Cohen E, Margalit I, Shochat T, Goldberg E \& Krause I. The relationship between the concentration of plasma homocysteine and chronic kidney disease: a cross sectional study of a large cohort. Journal of Nephrology 201932 783-789. (https://doi.org/10.1007/ s40620-019-00618-x)

17 Chuang CH, Lee YY, Sheu BF, Hsiao CT, Loke SS, Chen JC \& Li WC. Homocysteine and C-reactive protein as useful surrogate markers for evaluating CKD risk in adults. Kidney and Blood Pressure Research 2013 37 402-413. (https://doi.org/10.1159/000355722)

18 Francis ME, Eggers PW, Hostetter TH \& Briggs JP. Association between serum homocysteine and markers of impaired kidney function in adults in the United States. Kidney International 200466 303-312. (https://doi.org/10.1111/j.1523-1755.2004.00732.x)

19 Levey AS, Stevens LA, Schmid CH, Zhang YL, Castro AF, Feldman HI, Kusek JW, Eggers P, Van Lente F, Greene T, et al. A new equation to estimate glomerular filtration rate. Annals of Internal Medicine 2009 150 604-612. (https://doi.org/10.7326/0003-4819-150-9-20090505000006)
20 Vargas F, Moreno JM, Rodriguez-Gomez I, Wangensteen R, Osuna A, Alvarez-Guerra M \& Garcia-Estan J. Vascular and renal function in experimental thyroid disorders. European Journal of Endocrinology 2006154 197-212. (https://doi.org/10.1530/eje.1.02093)

21 Karanikas G, Schutz M, Szabo M, Becherer A, Wiesner K, Dudczak R \& Kletter K. Isotopic renal function studies in severe hypothyroidism and after thyroid hormone replacement therapy. American Journal of Nephrology 200424 41-45. (https://doi. org/10.1159/000075628)

22 Garibotto G, Sofia A, Valli A, Tarroni A, Di Martino M, Cappelli V, Aloisi F \& Procopio V. Causes of hyperhomocysteinemia in patients with chronic kidney diseases. Seminars in Nephrology 200626 3-7. (https://doi.org/10.1016/j.semnephrol.2005.06.002)

23 Galli F, Benedetti S, Buoncristiani U, Piroddi M, Conte C, Canestrari F, Buoncristiani E \& Floridi A. The effect of PMMA-based protein-leaking dialyzers on plasma homocysteine levels. Kidney International 200364 748-755. (https://doi.org/10.1046/j.15231755.2003.00134.x)

24 Bao L, Vlcek C, Paces V \& Kraus JP. Identification and tissue distribution of human cystathionine beta-synthase mRNA isoforms. Archives of Biochemistry and Biophysics 1998350 95-103. (https://doi. org/10.1006/abbi.1997.0486)

25 Selhub J. Homocysteine metabolism. Annual Review of Nutrition 1999 19 217-246. (https://doi.org/10.1146/annurev.nutr.19.1.217)

26 Garibotto G, Valli A, Anderstam B, Eriksson M, Suliman ME, Balbi M, Rollando D, Vigo E \& Lindholm B. The kidney is the major site of S-adenosylhomocysteine disposal in humans. Kidney International 200976 293-296. (https://doi.org/10.1038/ki.2009.117)

27 Chen YF, Li PL \& Zou AP. Effect of hyperhomocysteinemia on plasma or tissue adenosine levels and renal function. Circulation 2002106 1275-1281. (https://doi.org/10.1161/01. cir.0000027586.64231.1b)

28 Yi F, dos Santos EA, Xia M, Chen QZ, Li PL \& Li N. Podocyte injury and glomerulosclerosis in hyperhomocysteinemic rats. American Journal of Nephrology 200727 262-268. (https://doi. org/10.1159/000101471)

29 Long Y \& Nie J. Homocysteine in renal injury. Kidney Diseases 20162 80-87. (https://doi.org/10.1159/000444900)

30 Fox CS, Gona P, Larson MG, Selhub J, Tofler G, Hwang SJ, Meigs JB, Levy D, Wang TJ, Jacques PF, et al. A multi-marker approach to predict incident CKD and microalbuminuria. Journal of the American Society of Nephrology 201021 2143-2149. (https://doi.org/10.1681/ ASN.2010010085)

31 Ninomiya T, Kiyohara Y, Kubo M, Tanizaki Y, Tanaka K, Okubo K, Nakamura H, Hata J, Oishi Y, Kato I, et al. Hyperhomocysteinemia and the development of chronic kidney disease in a general population: the Hisayama study. American Journal of Kidney Diseases 200444 437-445. (doi:10.1016/S0272-6386(04)00813-3)

32 Levi A, Cohen E, Levi M, Goldberg E, Garty M \& Krause I. Elevated serum homocysteine is a predictor of accelerated decline in renal function and chronic kidney disease: A historical prospective study. European Journal of Internal Medicine 201425 951-955. (https://doi. org/10.1016/j.ejim.2014.10.014)

33 Xie D, Yuan Y, Guo J, Yang S, Xu X, Wang Q, Li Y, Qin X, Tang G, Huo $Y$, et al. Hyperhomocysteinemia predicts renal function decline: a prospective study in hypertensive adults. Scientific Reports 2015 516268. (https://doi.org/10.1038/srep16268)

34 Kong X, Ma X, Zhang C, Su H \& Xu D. Hyperhomocysteinemia increases the risk of chronic kidney disease in a Chinese middleaged and elderly population-based cohort. International Urology and Nephrology 201749 661-667. (https://doi.org/10.1007/s11255016-1452-3)

35 Xu X, Qin X, Li Y, Sun D, Wang J, Liang M, Wang B, Huo Y, Hou FF \& investigators of the Renal Substudy of the China Stroke Primary Prevention Trial (CSPPT). Efficacy of folic acid therapy on the progression of chronic kidney disease: the renal substudy of the 
China Stroke Primary Prevention Trial. Journal of American Medical Association Internal Medicine 2016176 1443-1450. (https://doi. org/10.1001/jamainternmed.2016.4687)

36 Jamison RL, Hartigan P, Kaufman JS, Goldfarb DS, Warren SR, Guarino PD, Gaziano JM \& Veterans Affairs Site Investigators. Effect of homocysteine lowering on mortality and vascular disease in advanced chronic kidney disease and end-stage renal disease: a randomized controlled trial. Journal of American Medical Association 2007298 1163-1170. (https://doi.org/10.1001/jama.298.10.1163)

37 House AA, Eliasziw M, Cattran DC, Churchill DN, Oliver MJ, Fine A, Dresser GK \& Spence JD. Effect of B-vitamin therapy on progression of diabetic nephropathy: a randomized controlled trial. Journal of American Medical Association 2010303 1603-1609. (https://doi. org/10.1001/jama.2010.490)

Received in final form 7 April 2021

Accepted 16 April 2021

Accepted Manuscript published online 16 April 2021
This work is licensed under a Creative Commons Attribution-NonCommercial 4.0 International License. 